\title{
Correction: Development of the food-based Lifelines Diet Score (LLDS) and its application in 129,369 Lifelines participants
}

\author{
Petra C. Vinke ${ }^{1}$ Eva Corpeleijn ${ }^{1} \cdot$ Louise H. Dekker ${ }^{2}$ - David R. Jacobs $\mathrm{Jr}^{3} \cdot$ Gerjan Navis $^{2}$ - Daan Kromhout ${ }^{1}$
}

Published online: 24 June 2019

(c) The Author(s), under exclusive licence to Springer Nature Limited 2019

\section{Correction to: European Journal of Clinical Nutrition https://doi.org/10.1038/s41430-018-0205-z published online 12 June 2018}

Since publication of the original paper, the authors discovered that due to a typographical error, the wrong lower cut point for acceptable energy intake/basal metabolic rate was given in the Methods section of the manuscript.
The published sentence was:

"A total of 14,726 participants with a ratio below 0.87 or above 2.75 were excluded $(<0.89$ or $>2.66$ for participants $>75$ years), leaving 129,369 participants in the study."

The correct sentence is:

"A total of 14,726 participants with a ratio below 0.50 (the 10th percentile in our cohort), or above 2.75 were excluded, leaving 129,369 participants in the study." 